\title{
RECONSTRUCTION OF THE THORACIC WALL WITH EPIPLOON AND ABDOMINAL LOWER DERMOCUTANEOUS RETAIL IN ANGIOSARCOMA MAMMARY
}

\author{
Darley Ferreira', Nancy Ferreira', Thais Ferreira \\ 1Department of Mastology and Reconstruction, Barão Lucena Hospital - Recife (PE), Brazil
}

Introduction: Breast angiosarcoma is a very rare and highly aggressive lesion, with an incidence of $0.5 \%$ to $1 \%$. Berg et al. recognized two groups of sarcoma: the first group includes malignant phylloid cysts, lymphomas, and hemangiosarcomas, and the second group includes stromal sarcomas, fibrosarcomas, leiomyosarcomas, histiocytomas, and giant cell sarcoma. Angiosarcomas are lesions of indefinite and friable masses, with a mean age of 35 years. Case Report: A 35-year-old man from Paulo Afonso-PE presents complaining of breast lump. He underwent tumorectomy and confirmed fibroadenoma and phylloids with atypia and mitosis. A battery of tests such as mammography (MMG)/ultrasonography (USG) confirmed the presence of a $1.5-\mathrm{cm}$ nodule in the breast. In addition, a new segmental resection surgery was performed, in which histopathological results confirmed a low-grade malignant phylloid cystosarcoma and demanding margins. The patient was proposed a new surgical of simple mastectomy with immediate reconstruction with silicone implant and latissimus dorsi flap. Finally, the surgery was performed and the histopathological result was the absence of residual neoplastic tissue, with an area of scar fibrosis and typical ductal hyperplasia. After recovery, the patient was referred to clinical oncology and radiotherapy, but both had no indication for adjuvant therapy. After 1 year, the patient returned to perform the symmetry of the opposite breast and reconstruction of the nipple-areola complex. In her follow-up, there were no changes in her examinations. After 2 years, she returned with a breast USG examination, which demonstrated an image nodular $1.5 \mathrm{~cm}$ adjacent to breast prosthesis and magnetic resonance imaging suggested the same image. A core was performed, confirming a recurrent malignant variant tumor. The tumor evolved very quickly, and the surgery was performed with an enlarged resection of the entire large and small pectoral and inclusion of the skin. For correction of the deformity, the rotation of the large epiploid with a lower abdominal dermocutaneous flap was used. Conclusion: The use of a technique with the large epiploid to cover the chest wall associated with a lower abdominal dermocutaneous flap presented a good alternative to correct chest wall deformity.

Keywords: Sarcoma; Breast Câncer; Breast Reconstruction. 